\title{
Bistable Helmholtz dark spatial optical solitons in materials with self-defocusing saturable nonlinearity
}

\author{
J. M. Christian and M. J. Lundie \\ Joule Physics Laboratory, \\ School of Computing, Science and Engineering, \\ University of Salford, Greater Manchester M5 4WT, United Kingdom \\ *j.christian@salford.ac.uk \\ Received (2 May 2017)
}

\begin{abstract}
We present, to the best of our knowledge, the first exact dark spatial solitons of a nonlinear Helmholtz equation with a self-defocusing saturable refractive-index model. These solutions capture oblique (arbitrary-angle) propagation in both the forward and backward directions, and they can also exhibit a bistability characteristic. A detailed derivation is presented, obtained by combining coordinate transformations and directintegration methods, and the corresponding solutions of paraxial theory are recovered asymptotically as a subset. Simulations examine the robustness of the new Helmholtz solitons, with stationary states emerging from a range of perturbed input beams.
\end{abstract}

Keywords: Helmholtz diffraction; saturable refractive index; spatial solitons

\section{Introduction}

Saturation is a universal phenomenon appearing throughout all of nonlinear science. Intuitively, one expects that no growth process in Nature can continue indefinitely: ultimately, a (system dependent) choking effect must always come into play to moderate the dynamics and so prevent unphysical 'run-away' (divergent) behaviour. In photonics, one is often concerned with potential saturation of the light-induced dielectric response of a particular optical material (either its refractive or absorptive properties). ${ }^{1,2}$ Well-known materials that exhibit saturation include some semiconductor-doped glasses (e.g., CdSSe and Schott OG 550 glass), ${ }^{3,4}$, iondoped crystals (e.g., $\mathrm{GdAlO}_{3}: \mathrm{Cr}^{3+}$ ),${ }^{5}$ bio-optical media, ${ }^{6} \pi$-conjugated polymers, ${ }^{7}$ and various photorefractive crystals (e.g., $\mathrm{LiNbO}_{3}$ and $\mathrm{SBN}$ ). ${ }^{8,9}$ The detailed mechanism underlying optical saturation is intricate, but at an atomic level it may be described phenomenologically in terms of the bleaching of excited states under high-intensity illumination. ${ }^{1,2}$

Many research groups have investigated optical phenomena connected to a saturable refractive index, and so a detailed survey of the literature is prohibitive here. However, one may readily identify key themes of investigation that are most closely allied to the problem of spatial soliton propagation: paraxial ${ }^{10-13}$ and ultranarrow ${ }^{14-16}$ nonlinear beams, and (because of the structure of their governing equa- 
tions) conventional pulse propagation models. ${ }^{17-19}$ Other related photonics-based contexts where saturation effects can become important include cavity, multi-hump, photorefractive and incoherent solitons, soliton clusters and vortices, spontaneous pattern formation, and quantum-optical squeezing.

Here, we are concerned with dark spatial optical solitons propagating in twodimensional (2D) uniform planar waveguides. ${ }^{20,21}$ The core material is assumed to have a self-defocusing saturable refractive index of the form ${ }^{22-24}$

$$
n_{\mathrm{NL}}(I)=-\frac{n_{2} I_{\mathrm{sat}}}{2}\left[1-\frac{1}{\left(1+I / I_{\mathrm{sat}}\right)^{2}}\right],
$$

where $n_{2}$ is taken to be a positive parameter throughout (it can be interpreted as the effective Kerr coefficient for sufficiently low-level illumination), $I$ denotes intensity and $I_{\text {sat }}$ is a constant. In the limit that $I / I_{\text {sat }} \ll \mathcal{O}(1)$, it follows that $n_{\mathrm{NL}}(I) \simeq-n_{2} I\left[1-(3 / 2)\left(I / I_{\text {sat }}\right)\right]$ (i.e., self-defocusing Kerr-type with a small correction) while $n_{\mathrm{NL}}$ flattens out to the fixed value $-n_{2} I_{\text {sat }} / 2$ when $I / I_{\text {sat }} \gg \mathcal{O}(1)$. While the use of an apparently complicated form may seem undesirable initially, it turns out that propagation equations with that type of nonlinearity may be integrated exactly to yield implicit soliton solutions. ${ }^{22,23}$ In contrast, much simpler algebraic models such as $n_{\mathrm{NL}}(I)= \pm n_{2} I /\left(1+I / I_{\text {sat }}\right)$ tend not to support exact solutions rendering analyses of those systems and their stationary states predominantly numerical. ${ }^{17-19}$ Generic models of saturation are important because, while one may be able to capture leading-order effects through competing-polynomial nonlinearities, any finite power series expansion in $I / I_{\text {sat }}$ will eventually fail for sufficiently strong illumination.

The layout of this paper is as follows. Section 2 considers some of the more recent developments in optical-soliton literature, while in Sec. 3 we address the Helmholtz propagation problem and highlight important distinctions between our approach and those found in other works. The governing equation is proposed and its plane wave solutions are explored in detail. In Sec. 4, exact dark solitons are sought by integrating the coupled intensity-phase quadrature equations. The spatial asymptotics of these solitons are mapped onto a plane-wave field using similar techniques to those established in Ref. 25, and geometrical transformations ${ }^{26}$ are deployed to obtain more general off-axis solutions. A bistability characteristic is also discussed. ${ }^{17-19,22,23,27}$ Further analysis in Sec. 5 demonstrates that Helmholtz Kerr dark solitons ${ }^{28}$ emerge from the new solutions in the limit that saturation effects are negligible (such an asymptotic result is a physical and mathematical requirement). Moreover, in an appropriate multiple limit corresponding to the slowly-varying envelope approximation (SVEA), the classic paraxial dark soliton ${ }^{23}$ is recovered. Simulations test the stability of Helmholtz saturable dark solitons in Sec. 6, where their role as potentially robust attractors is investigated. We conclude, in Sec. 7, with some remarks about the power and wider applicability of theoretical formulations based on Helmholtz diffraction. 


\section{Recent Developments for Optical Solitons}

Soliton-type excitations underpin many aspects of nonlinear optics, where the interplay between intense laser light and increasingly-sophisticated material response functions give rise to novel emergent phenomena. With models bound by the SVEA, ensuing Schrödinger classes of governing equation lend themselves well to the development of new solution methods (aided by symbolic-algebra software). ${ }^{29}$ The traditional nonlinearities which admit exact solutions - cubic, power-law, dual powerlaw, parabolic, and logarithmic - continue to drive modern research innovations. For instance, they have played key roles in generalizing analyses to regimes involving pulse propagation in metamaterial contexts. Kerr-law systems with a suite of higher-order derivative terms are now known support additional exotic solutions such as cosh-Gaussian and sech-tanh stationary states obtained through a semiinverse variational principle. ${ }^{30}$ These same fundamental nonlinearities are also pertinent to the design of novel twin- and multi-core nonlinear directional couplers, where the $G^{\prime} / G$-expansion method has been used for seeking dark solitons. ${ }^{31}$

Consideration of pulse trains in bimodal optical fibre systems with longitudinally patterned cores have led to the discovery of coupled cnoidal waves (periodic solutions described by Jacobi elliptic functions) and self-compression effects in some parameter regimes of generalized Manakov-type systems. ${ }^{32}$ Nonlinear Schrödinger (NLS) equations accounting for a slowly-varying (in time) component of the thirdorder polarization and a delayed Raman response predict the self-steepening and self-frequency shifting, respectively, of travelling waves. When third-order dispersion is negligible, exact solitons may exhibit either super- or sub-luminal propagation characteristics and chirality. ${ }^{33}$

In the spatial domain, similarity-reduction techniques ${ }^{34}$ have recently found that a cubic-quintic NLS model with variable coefficients may support families of bright and dark similaritons (localized waveforms that expand on propagation but nevertheless preserve their shape with respect to a particular scaling law) in waveguides with a transversely- (graded) and longitudinally- (tapered) modulated refractive-index profile. New types of single- and two-component electro-optic gray solitons in two-photon photorefractive materials, where the valence and conduction bands are typically separated by an intermediate energy level, have also been calculated numerically. ${ }^{35}$

Finally, liquid crystals provide another arena in which to generate and manipulate nonlinear-optical waves through their molecular reorientational properties and the nonlocal nature of the nematic phase. Observed excitations include (bright and dark) solitons and nematicons, ${ }^{36}$ along with wider classes of distributed solutions (shock, snoidal, and singular waves) recently derived with the extended trial equation method. ${ }^{37}$ Discrete nematic liquid-crystal waveguides have also been shown to support a diverse range of phenomena, including dispersive shockwaves and oscillatory structures (e.g., shelf-like breathers), that are dependent upon the strength of intersite coupling (that is, on nonlocality). ${ }^{38}$ 


\section{Helmholtz Diffraction}

\subsection{Nonlinear Helmholtz equations}

Formulations of traditional beam-propagation problems based on Helmholtz equations differ fundamentally from those of their paraxial (NLS-type) counterparts. ${ }^{39}$ While the experimental geometry of scalar wave optics may be a common feature of both approaches - typically a transverse electric (TE-polarized) field operating in the continuous-wave $(\mathrm{cw})$ regime, and where beam waists are much longer than the wavelength - the former does not assume slowly-varying envelopes. This distinction makes the Helmholtz level of description elliptical in nature, and thus fully second-order-symmetric in the spatial coordinates. In contrast, paraxial diffraction equations tend to be parabolic and thus easier to solve mathematically and computationally. More subtly, the symmetric nature of nonlinear Helmholtz equations can often rule out the existence of exact solutions that are analogues of those commonly found in paraxial theory (where standard solution methods can start to break down ${ }^{28,40}$ ). Spatial symmetry, then, has to be built into any analytical approach from the outset (e.g., solution decomposition and reduced equations).

Previously, we have considered bright spatial solitons of a Helmholtz equation with the focusing version of model (1.1). ${ }^{41}$ By deploying such a nonparaxial framework, the angular limitations of paraxial diffraction - rooted in the SVEA - may be lifted completely. Helmholtz soliton theory then provides a compact and elegant mathematical formalism for modelling oblique-evolution properties of scalar beams at arbitrary angles (in the laboratory frame) with respect to the longitudinal reference direction. ${ }^{26}$ Indeed, off-axis considerations are elementary for even the simplest configurations: (i) interacting beams ${ }^{40}$ and (ii) reflection / refraction of bright ${ }^{42,43}$ and dark ${ }^{44,45}$ solitons at interfaces between dissimilar materials. The Helmholtz angular type of nonparaxiality thus underpins, in principle, almost every conceivable optical device design and architecture.

Here we analyze the dark spatial soliton counterparts to the bright solutions reported in Ref. 41. Despite satisfying a more complicated governing equation than their simpler (paraxial) variants, they retain the same physical structure (namely a plane wave background field across which an obliquely-propagating stationary gray 'dip' may travel). ${ }^{20,21}$ The dark solitons studied here are non-trivial spatially-symmetric generalizations of the classic solutions derived by Krolikowski and Luther-Davies. ${ }^{23}$ We have thus derived convenient nonlinear basis functions for analyzing dark beams in a wide range of arbitrary-angle scenarios, and where the description of a material's optical response goes beyond the traditional cubic ${ }^{28}$ and cubic-quintic ${ }^{25}$ power-series idealizations.

\subsection{Field and envelope equations}

We are interested in the off-axis properties of broad optical beams where the waist $w_{0}$ is much greater than the free-space carrier wavelength $\lambda$. Since the inequality 
$\epsilon \equiv \lambda / w_{0} \ll \mathcal{O}(1)$ is always fully respected, ultranarrow-beam corrections (obtained from $\epsilon$-type order-of-magnitude analyses of Maxwell's equations) are unnecessary. ${ }^{46,47}$ Hence, the polarization-scrambling term $\nabla(\nabla \cdot \mathbf{E})$ can be safely neglected $^{14-16,48,49}$ and attention is paid exclusively to scalar diffraction. ${ }^{26,41} \mathrm{We}$ consider a TE-polarized cw beam, as represented by

$$
\tilde{E}(x, z, t)=E(x, z) \exp (-i \omega t)+E^{*}(x, z) \exp (+i \omega t) .
$$

The laboratory space and time coordinates are denoted by $(x, z)$ and $t$, respectively, and $\omega$ is the optical frequency. If the transverse spatial variations of the beam are sufficiently slow on the scalelength of the underlying carrier wavelength, the field $E(x, z)$ satisfies the 2D Helmholtz equation ${ }^{26,50-52}$

$$
\left(\frac{\partial^{2}}{\partial z^{2}}+\frac{\partial^{2}}{\partial x^{2}}\right) E(x, z)+\frac{\omega^{2} n^{2}}{c^{2}} E(x, z)=0,
$$

where $c$ is the vacuum speed of light. Spatial symmetry appears in Eq. (3.2) through the equivalent status of the $x$ and $z$ derivatives in the in-plane Laplacian $\partial_{z z}+$ $\partial_{x x}$ (where $\partial_{x} \equiv \partial / \partial x$, etc.) so that diffraction occurs in both these dimensions. Nonlinearity enters the propagation problem through the refractive index $n \equiv n_{0}+$ $n_{\mathrm{NL}}(I)$, where $n_{0}$ is the linear index at frequency $\omega$ and $I(x, z) \equiv|E(x, z)|^{2}$ is the (local) light intensity. For a weak nonlinear response, where $\left|n_{\mathrm{NL}}\right| \ll n_{0}$, one has that $n^{2}(I) \simeq n_{0}^{2}+2 n_{0} n_{\mathrm{NL}}(I)$ to an excellent approximation.

The reference direction is chosen to be $z$ by representing $E$ through the decomposition $E(x, z)=E_{0} u(x, z) \exp (i k z)$, where the real constant $E_{0}$ scales the field strength, $u(x, z)$ is the dimensionless envelope, and $k \equiv(\omega / c) n_{0}=(2 \pi / \lambda) n_{0}$. The following governing equation for $u$, incorporating optical nonlinearity (1.1), can now be derived without further approximation:

$$
\kappa \frac{\partial^{2} u}{\partial \zeta^{2}}+i \frac{\partial u}{\partial \zeta}+\frac{1}{2} \frac{\partial^{2} u}{\partial \xi^{2}}-\frac{1}{2} \frac{2+\gamma|u|^{2}}{\left(1+\gamma|u|^{2}\right)^{2}}|u|^{2} u=0 .
$$

The normalized spatial coordinates are $\zeta=z / L_{D}$ and $\xi=2^{1 / 2} x / w_{0}$, where $L_{D}=k w_{0}^{2} / 2$ is the diffraction length of a reference (paraxial) Gaussian beam with waist $w_{0}$. The inverse beam width is quantified by $\kappa \equiv 1 /\left(k w_{0}\right)^{2}=\epsilon^{2} / 4 \pi^{2} n_{0}^{2} \ll$ $\mathcal{O}(1)$. The units of electric field amplitude are determined by the choice of scaling $E_{0}^{2} n_{2} k L_{D} / n_{0} \equiv 1$, while $\gamma^{-1} \equiv I_{\text {sat }} / E_{0}^{2}$ is the normalized saturation intensity. We stress that full spatial symmetry is preserved when transforming between models (3.2) and (3.3) because the assumption of a slowly-varying envelope, that is to say $\kappa\left|\partial_{\zeta \zeta} u\right| \ll\left|\partial_{\zeta} u\right|$, has not been made.

\subsection{Plane waves}

The plane waves of Eq. (3.3) can be obtained by seeking solutions of the form

$$
u(\xi, \zeta)=\rho_{0}^{1 / 2} \exp \left[i\left(k_{\xi} \xi+k_{\zeta} \zeta\right)\right] \exp \left(-i \frac{\zeta}{2 \kappa}\right),
$$




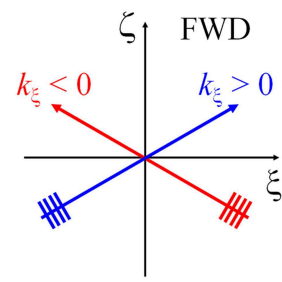

(a)

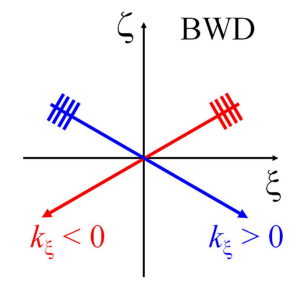

(b)

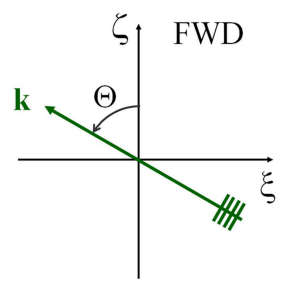

(c)

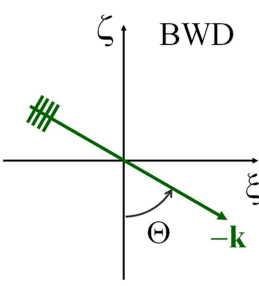

(d)

Fig. 1. Schematic representation of plane waves in scaled units. (a) Forward (FWD) and (b) backward (BWD) solutions described by $k_{\zeta}>0$ and $k_{\zeta}<0$, respectively, according to Eq. (3.4d). A more convenient representation, with wavevector $\mathbf{k} \equiv\left(k_{\xi}, k_{\zeta}\right)$ given by Eq. (3.6), introduces a transverse velocity parameter $V$, which is connected to angle $\Theta$ through $V=\tan \Theta$. Panes (c) and (d) consider FWD and BWD waves, respectively, when $V>0$.

where $\rho_{0} \equiv|u|^{2}$ is the (uniform) light intensity and $\mathbf{k} \equiv\left(k_{\xi}, k_{\zeta}\right)$ is the wavevector. Substitution of Eq. (3.4a) into Eq. (3.3) then yields the elliptic dispersion relation

$$
\kappa k_{\zeta}^{2}-\frac{1}{4 \kappa}+\frac{k_{\xi}^{2}}{2}=\beta
$$

where the full significance of the parameter

$$
\beta \equiv-\left(\frac{\rho_{0}}{2}\right) \frac{2+\gamma \rho_{0}}{\left(1+\gamma \rho_{0}\right)^{2}}
$$

will become clear in Sec. 4 . One can obtain $k_{\zeta}$ from Eq. (3.4b), recognizing that there must always be two distinct solution branches due to the fully-quadratic (in both $x$ and $z$ ) nature of scalar Helmholtz diffraction:

$$
k_{\zeta}= \pm \frac{1}{2 \kappa}\left[1+4 \kappa\left(\beta-\frac{k_{\xi}^{2}}{2}\right)\right]^{1 / 2} .
$$

For fixed transverse spatial frequency $k_{\xi}$, there exist waves that travel in both forward (in our convention, always the upper sign) and backward (lower sign) longitudinal senses (see Figs. 1(a) and 1(b)).

For propagating waves (i.e., those solutions that are non-evanescent in $\zeta$ ), there clearly exists an upper limit on the value of $\left|k_{\xi}\right|$, since

$$
\left(k_{\xi}^{2}\right)_{\max }=\frac{1}{2 \kappa}\left[1-\left(\frac{2 \kappa}{\gamma}\right) \frac{2+\gamma \rho_{0}}{\left(1+\gamma \rho_{0}\right)^{2}} \gamma \rho_{0}\right]
$$

cannot be negative. The maximum value of $\left|k_{\xi}\right|$ corresponds to regimes where $k_{\zeta}=$ 0 , and hence describes waves travelling perpendicularly to the reference direction (i.e., in the $\xi$ direction). An equivalent interpretation is that Eq. (3.5a) determines a boundary in Fourier space between forward and backward propagation. ${ }^{53,54}$

Equation (3.5a) yields two interesting results related to the quantity $2 \kappa \gamma^{-1}$, which quantifies the interplay between beam nonparaxiality (through the parameter $\kappa$ ) and saturation (as determined by $\gamma^{-1}$ ). When $\gamma^{-1} \leq 1 / 2 \kappa$, the intensity $\rho_{0}$ can 
assume (in principle) any value without violating geometrical constraints. Similarly, $\gamma^{-1}>1 / 2 \kappa$ requires a cut-off such that

$$
\rho_{0}<\gamma^{-1}\left[\sqrt{\frac{2 \kappa \gamma^{-1}}{2 \kappa \gamma^{-1}-1}}-1\right] .
$$

Since $\kappa \ll \mathcal{O}(1)$ and intensities should retain moderate values (in order to ensure the validity of scalar modelling), inequality (3.5b) can be safely discarded. We note, in passing, that $\kappa$-dependent inequalities determining plane-wave characteristics have also been reported for cubic ${ }^{28}$ and cubic-quintic ${ }^{25}$ Helmholtz equations.

An alternative and often more convenient approach is to associate a conventional transverse velocity parameter $V$ with $k_{\xi} \cdot{ }^{25}$ In that representation, one may construct $\mathbf{k}$ as

$$
\mathbf{k} \equiv\left(k_{\xi}, k_{\zeta}\right)= \pm \sqrt{\frac{1+4 \kappa \beta}{1+2 \kappa V^{2}}}\left(-V, \frac{1}{2 \kappa}\right),
$$

so that switching between the \pm sign in Eq. (3.6) simply reverses the entire wave vector (i.e., $\mathbf{k} \rightarrow-\mathbf{k}$ ) rather than just its longitudinal projection. Hence, one may now appreciate that there is no physical distinction between forward and backward waves (the pair of solutions are connected by a $180^{\circ}$ rotation of the observer's coordinate axes or, equivalently, a reversal of k) (see Figs. 1(c) and 1(d)). The propagation angle $\Theta$ with respect to the $\zeta$ axis is now given by $\tan \Theta \equiv V$.

The difference between propagation problems described within scaled and unscaled units is perhaps most powerfully demonstrated through angular considerations, where the connection between propagation angle $\theta$ in $(x, z)$ coordinates and transverse velocity $V$ in $(\xi, \zeta)$ coordinates is given by ${ }^{26}$

$$
\tan \theta=\sqrt{2 \kappa} V .
$$

Equation (3.7) plays a fundamental role in Helmholtz soliton theory and throughout the subsequent analysis here. It shows that even when $\kappa \ll \mathcal{O}(1)$ (as is the case for broad scalar beams), the geometrical factor $2 \kappa V^{2}$ correcting paraxial theory can become arbitrarily large as $|\theta| \rightarrow 90^{\circ}$.

\subsection{Modulational stability}

By deploying the technique developed in Ref. 53, one can analyze the stability of plane waves against small-amplitude modulations. Without loss of generality, we consider an on-axis wave and seek perturbed solutions to Eq. (3.3) of the form

$$
u(\xi, \zeta)=\rho_{0}^{1 / 2}[1+\mu a(\xi, \zeta)] \exp \left(i k_{\zeta} \zeta\right) \exp \left(-i \frac{\zeta}{2 \kappa}\right) .
$$

Here, $a$ is a complex perturbation of $\mathcal{O}(1)$ magnitude that describes disturbances to both amplitude and phase of the underlying wave, while $\mu$ is a formal expansion parameter satisfying $\mu \ll \mathcal{O}(1)$. By substituting Eq. (3.8a) into Eq. (3.3), cancelling 
the plane wave background (using dispersion relation (3.4b)) and isolating terms at $\mathcal{O}(\mu)$, one can show that $a$ satisfies the linearized Helmholtz equation

$$
\kappa \frac{\partial^{2} a}{\partial \zeta^{2}} \pm i \sqrt{1+4 \kappa \beta} \frac{\partial a}{\partial \zeta}+\frac{1}{2} \frac{\partial^{2} a}{\partial \xi^{2}}-\frac{\rho_{0}}{\left(1+\gamma \rho_{0}\right)^{3}}\left(a+a^{*}\right)=0 .
$$

Equation (3.8b) admits single Fourier mode solutions of the form

$$
a(\xi, \zeta)=a_{1} \exp \left[i\left(K_{\xi} \xi+K_{\zeta} \zeta\right)\right]+a_{2}^{*} \exp \left[-i\left(K_{\xi} \xi+K_{\zeta}^{*} \zeta\right)\right],
$$

where $a_{1}$ and $a_{2}$ are complex constants. The perturbation has a transverse spatial frequency $K_{\xi}$ (and hence a pattern scalelength of $2 \pi / K_{\xi}$ ) and propagation constant $K_{\zeta}$ (which must be purely real in order to avoid the longitudinal growth of $a$ ).

After some algebra, it can be shown that the perturbation dispersion relation connecting $K_{\xi}$ and $K_{\zeta}$ is given by the quartic equation

$$
\kappa^{2} K_{\zeta}^{4}-\left[1+4 \kappa \beta-2 \kappa \frac{K_{\xi}^{2}}{2}-\frac{2 \kappa \rho_{0}}{\left(1+\gamma \rho_{0}\right)^{3}}\right] K_{\zeta}^{2}+\frac{K_{\xi}^{2}}{2}\left[\frac{K_{\xi}^{2}}{2}+\frac{2 \rho_{0}}{\left(1+\gamma \rho_{0}\right)^{3}}\right]=0 .
$$

Equation (3.8d) can be solved exactly for $K_{\zeta}$, whereupon one finds four roots. Crucially, there is no long-wave instability since on all four solution branches, $\Im m\left(K_{\zeta}\right)=0$ in small- $K_{\xi}$ regimes. Hence, linear analysis predicts that the plane waves of Eq. (3.3) are robust against small-amplitude fluctuations (a key ingredient for establishing the potential stability of dark solitons). That prediction is fully supported by numerical computations, which have uncovered no evidence of long-wavelength spontaneous modulational instability.

\section{Helmholtz Dark Solitons}

\subsection{Solution decomposition $\&$ quadrature equations}

To proceed with finding exact analytical solitons, one can split $u$ into its intensity and phase quadratures, denoted by $\rho(\xi, \zeta)$ and $\Psi(\xi, \zeta)$, respectively, according to

$$
u(\xi, \zeta)=\rho^{1 / 2}(\xi, \zeta) \exp [i \Psi(\xi, \zeta)] \exp \left(-i \frac{\zeta}{2 \kappa}\right) .
$$

By substituting desired solution (4.1a) into Eq. (3.3), a quite general pair of coupled quadrature equations can be obtained which $\rho$ and $\Psi$ must satisfy. The real part of Eq. (3.3) leads to

$$
\begin{aligned}
& \frac{2}{\rho}\left(\frac{\partial^{2} \rho}{\partial \xi^{2}}+2 \kappa \frac{\partial^{2} \rho}{\partial \zeta^{2}}\right)-\frac{1}{\rho^{2}}\left[\left(\frac{\partial \rho}{\partial \xi}\right)^{2}+2 \kappa\left(\frac{\partial \rho}{\partial \zeta}\right)^{2}\right] \\
& -4\left[\left(\frac{\partial \Psi}{\partial \xi}\right)^{2}+2 \kappa\left(\frac{\partial \Psi}{\partial \zeta}\right)^{2}\right]=8\left[\left(\frac{\rho}{2}\right) \frac{2+\gamma \rho}{\left(1+\gamma \rho_{0}\right)^{2}}-\frac{1}{4 \kappa}\right]
\end{aligned}
$$




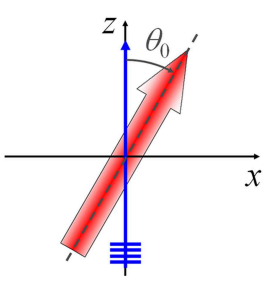

(a)

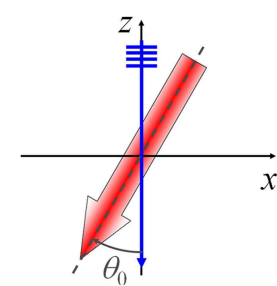

(b)

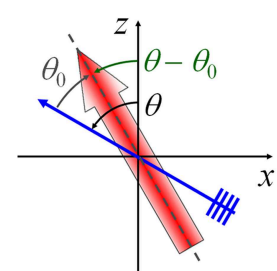

(c)

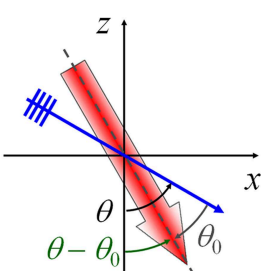

(d)

Fig. 2. Schematic representation of dark spatial soliton geometry in the $(x, z)$ laboratory frame. (a) Forward and (b) backward solutions comprising an on-axis plane wave and an off-axis gray dip travelling at angle $\theta_{0}$ relative to the plane wave background. Most general (c) forward and (d) backward solutions. The background field travels off-axis at angle $\theta$, while relative angle $\theta_{0}$ remains unchanged. The dip thus makes angle $\theta-\theta_{0}$ with respect to the longitudinal direction.

while the imaginary part yields

$$
\rho\left(\frac{\partial^{2} \Psi}{\partial \xi^{2}}+2 \kappa \frac{\partial^{2} \Psi}{\partial \zeta^{2}}\right)+\frac{\partial \rho}{\partial \xi} \frac{\partial \Psi}{\partial \xi}+2 \kappa \frac{\partial \rho}{\partial \zeta} \frac{\partial \Psi}{\partial \zeta}=0 .
$$

By factoring out the rapidly-varying carrier-wave phase factor $\exp (-i \zeta / 2 \kappa) \equiv$ $\exp (-i k z)$ in decomposition (4.1a), explicit spatial symmetry has been restored to quadrature equations (4.1b) and (4.1c), which are much more complicated than their counterparts in paraxial theory. However, they can still be integrated to find exact analytical dark solitons if one exploits the physical structure of such solutions (i.e., a uniform background modulated by a phase-topological gray 'dip').

\subsection{Mapping onto plane waves}

We begin by considering a particular case: where the uniform background wave is propagating on-axis (i.e., with zero transverse velocity), while the gray dip travels obliquely to the $\zeta$ axis with intrinsic velocity $V_{0}$ (see Figs. 2(a) and 2(b)). Such a solution is prescribed by $\Psi(\xi, \zeta)=\Psi_{\text {sol }}(\xi, \zeta)+k_{\zeta} \zeta$, where $\Psi_{\text {sol }}(\xi, \zeta)$ is the soliton phase distribution and $k_{\zeta}$ is the plane wave propagation constant (obtained from Eq. (3.6) by setting $V=0$ ). Since $\partial_{\xi} \Psi=\partial_{\xi} \Psi_{\text {sol }}, \partial_{\xi \xi} \Psi=\partial_{\xi \xi} \Psi_{\text {sol }}, \partial_{\zeta} \Psi=\partial_{\zeta} \Psi_{\text {sol }}+k_{\zeta}$, and $\partial_{\zeta \zeta} \Psi=\partial_{\zeta \zeta} \Psi_{\text {sol }}$, it follows that Eqs. (4.1b) and (4.1c) become

$$
\begin{aligned}
& \frac{2}{\rho}\left(\frac{\partial^{2} \rho}{\partial \xi^{2}}+2 \kappa \frac{\partial^{2} \rho}{\partial \zeta^{2}}\right)-\frac{1}{\rho^{2}}\left[\left(\frac{\partial \rho}{\partial \xi}\right)^{2}+2 \kappa\left(\frac{\partial \rho}{\partial \zeta}\right)^{2}\right]-4\left[\left(\frac{\partial \Psi_{\text {sol }}}{\partial \xi}\right)^{2}+2 \kappa\left(\frac{\partial \Psi_{\text {sol }}}{\partial \zeta}\right)^{2}\right] \\
& \quad=8\left[\left(\frac{\rho}{2}\right) \frac{2+\gamma \rho_{0}}{\left(1+\gamma \rho_{0}\right)^{2}}+2 \kappa k_{\zeta} \frac{\partial \Psi_{\text {sol }}}{\partial \zeta}+\left(\kappa k_{\zeta}^{2}-\frac{1}{4 \kappa}\right)\right]
\end{aligned}
$$

and

$$
\rho\left(\frac{\partial^{2} \Psi_{\mathrm{sol}}}{\partial \xi^{2}}+2 \kappa \frac{\partial^{2} \Psi_{\mathrm{sol}}}{\partial \zeta^{2}}\right)+\frac{\partial \rho}{\partial \xi} \frac{\partial \Psi_{\mathrm{sol}}}{\partial \xi}+2 \kappa \frac{\partial \rho}{\partial \zeta} \frac{\partial \Psi_{\mathrm{sol}}}{\partial \zeta}+2 \kappa k_{\zeta} \frac{\partial \rho}{\partial \zeta}=0,
$$

respectively. By noting that the soliton intensity and phase distributions move along 
the characteristic $\xi-V_{0} \zeta=0$, it is instructive to define a new variable,

$$
s \equiv \frac{\xi-V_{0} \zeta}{\sqrt{1+2 \kappa V_{0}^{2}}},
$$

which represents the coordinate perpendicular to the propagation direction. Under transformation (4.3a), the operators $\partial_{\xi}$ and $\partial_{\zeta}$ may be replaced according to

$$
\frac{\partial}{\partial \xi}=\frac{1}{\sqrt{1+2 \kappa V_{0}^{2}}} \frac{d}{d s} \text { and } \frac{\partial}{\partial \zeta}=-\frac{V_{0}}{\sqrt{1+2 \kappa V_{0}^{2}}} \frac{d}{d s} .
$$

Equation $(4.2 \mathrm{~b})$ can be recast into the more compact form

$$
\frac{d}{d s}\left[\rho \frac{d \Psi_{\mathrm{sol}}}{d s} \mp V_{0}\left(\frac{1+4 \kappa \beta}{1+2 \kappa V_{0}^{2}}\right)^{1 / 2} \rho\right]=0,
$$

which, after a single integration with respect to $s$, yields

$$
\frac{d \Psi_{\mathrm{sol}}}{d s}=\frac{c_{1}}{\rho} \pm V_{0}\left(\frac{1+4 \kappa \beta}{1+2 \kappa V_{0}^{2}}\right)^{1 / 2},
$$

where $c_{1}$ is a constant to be determined. By applying the same coordinate change to Eq. (4.2a) and eliminating the phase derivative $d_{s} \Psi_{\text {sol }}$ by deploying Eq. (4.4b), it can be shown that $\rho$ must satisfy

$$
\frac{d}{d \rho}\left[\frac{1}{\rho}\left(\frac{d \rho}{d s}\right)^{2}\right]=8\left(\frac{\rho}{2}\right) \frac{2+\gamma \rho}{(1+\gamma \rho)^{2}}+4 \frac{c_{1}^{2}}{\rho^{2}}+8 \chi .
$$

The constant $\chi$ appearing in Eq. (4.5a), defined as

$$
\chi \equiv \frac{\kappa k_{\zeta}^{2}}{1+2 \kappa V_{0}^{2}}-\frac{1}{4 \kappa},
$$

wraps up all the information about off-axis propagation into a single parameter. Hence, when written in terms of $s$, the Helmholtz and paraxial quadrature equations are seen to be formally identical to each other (a physical requirement).

\subsection{Boundary conditions}

Before further analysis, the asymptotic behaviour of the quadratures $\rho(s)$ and $\Psi_{\text {sol }}(s)$ needs to be considered. For dark soliton solutions, one tends to place restrictions on $\rho$ according to

$$
\lim _{s \rightarrow \pm \infty} \rho(s) \equiv \rho_{0} \quad \text { and } \quad \lim _{s \rightarrow 0} \rho(s) \equiv \rho_{1},
$$

where $\rho_{1}$ is the intensity at the beam centre, $\rho_{0}$ is the intensity of the plane wave background field, and $0<\rho_{1} \leq \rho_{0}$. Derivatives of $\rho$ and $\Psi_{\text {sol }}$ must also vanish at spatial infinity (where $u$ becomes a plane wave):

$$
\lim _{s \rightarrow \pm \infty} \frac{d}{d s} \rho(s)=0 \quad \text { and } \quad \lim _{s \rightarrow \pm \infty} \frac{d}{d s} \Psi_{\text {sol }}(s)=0 .
$$


Applying boundary conditions (4.6a) and (4.6b) to Eq. (4.4b) shows that the constant $c_{1}$ and the intrinsic velocity $V_{0}$ are connected thus:

$$
\frac{c_{1}}{\rho_{0}}=\mp V_{0}\left(\frac{1+4 \kappa \beta}{1+2 \kappa V_{0}^{2}}\right)^{1 / 2},
$$

capturing both forward- and backward-travelling configurations. Moreover, the sign of $c_{1}$ depends upon the interplay between the sign of $V_{0}$ and also the longitudinal sense of propagation (a fact that turns out to be crucial for determining the soliton phase distribution). For instance, the forward solution with a positive $V_{0}$ corresponds to the configuration shown in Fig. 2(a) and hence $c_{1}$ must be negative.

\subsection{Intensity $\&$ phase quadratures}

To proceed with finding the intensity distribution, we perform a direct integration of Eq. (4.5a) to yield

$$
\left(\frac{d \rho}{d s}\right)^{2}=8 \chi \rho^{2}+\frac{4}{\gamma^{2}}\left(\gamma \rho+\frac{1}{1+\gamma \rho}\right) \rho-4 c_{1}^{2}+c_{2} \rho,
$$

where $c_{2}$ is another constant. In expediting the second integration, it is instructive to express Eq. (4.7a) in the factorized form

$$
\left(\frac{d \rho}{d s}\right)^{2}=4 D \frac{\left(\rho-\rho_{1}\right)\left(\rho_{0}-\rho\right)^{2}}{(1+\gamma \rho)},
$$

noting that $\rho_{0}$ is a double root, $\rho_{1}$ is a single root, and $D$ is a constant to be determined. After some algebraic manipulations comparing Eqs. (4.7b) and (4.7a), it can be shown that $c_{1}, c_{2}$ and $\chi$ are obtained from

$$
\begin{aligned}
\frac{c_{1}^{2}}{\rho_{0}^{2}} & =D \rho_{1}, \\
\frac{c_{2}}{4} & =\left[\rho_{0}^{2}\left(1+\gamma \rho_{1}\right)+2 \rho_{0} \rho_{1}\right] D-\frac{1}{\gamma^{2}}, \\
\chi & =\frac{1}{2 \gamma}(D-1) .
\end{aligned}
$$

All three constants and parametrized by $D \equiv\left(1+\gamma \rho_{1}\right)^{-1}\left(1+\gamma \rho_{0}\right)^{-2}$.

Equation $(4.7 \mathrm{~b})$ can now be separated and integrated, remembering that dark solutions satisfy $d \rho / d s>0$ in the domain $0<s<+\infty$. That procedure leads to an implicit equation for the intensity distribution $\rho(s)$ which, in the representation of Krolikowski and Luther-Davies, ${ }^{23}$ is

$$
\left(\frac{\rho_{0}+\gamma^{-1}}{\rho_{0}-\rho_{1}}\right)^{1 / 2} \tanh ^{-1}\left[\left(\frac{\rho_{0}+\gamma^{-1}}{\rho_{0}-\rho_{1}}\right)^{1 / 2} \eta\right]-\tanh ^{-1}(\eta)=\frac{\gamma^{-1 / 2} s}{\left(1+\gamma \rho_{0}\right)\left(1+\gamma \rho_{1}\right)^{1 / 2}} .
$$


Here, the (non-negative) functional $\eta[\rho]$ is defined as

$$
\eta[\rho] \equiv\left(\frac{\rho-\rho_{1}}{\rho+\gamma^{-1}}\right)^{1 / 2},
$$

so that $\eta(s) \equiv \eta[\rho(s)]$. An explicit integral representation for $\Psi_{\text {sol }}$ can be obtained by combining Eq. (4.4b) with relation (4.6c) so that

$$
\frac{d \Psi_{\mathrm{sol}}}{d s}=\left(\frac{c_{1}}{\rho_{0}}\right)\left(\frac{\rho_{0}-\rho}{\rho}\right) .
$$

After a straightforward transformation, Eq. (4.9a) can be integrated with respect to $\rho$ (rather than $s$ ), such that in the domain $s>0$, we have that

$$
\Psi_{\mathrm{sol}}[\rho]=\mp \frac{1}{2} F\left(\gamma \rho_{0}\right)^{1 / 2} \int \frac{d \rho}{\rho}\left(\frac{\rho+\gamma^{-1}}{\rho-\rho_{1}}\right)^{1 / 2} .
$$

For convenience, the standard contrast parameter, $F^{2} \equiv \rho_{1} / \rho_{0}$, has been introduced and where $0<F^{2} \leq 1$ captures grayness of the solution. Evaluating the integral in Eq. (4.9b) yields the exact expression

$$
\Psi_{\mathrm{sol}}[\rho]=\mp\left\{\tan ^{-1}\left[\frac{\eta(\rho)}{F\left(\gamma \rho_{0}\right)^{1 / 2}}\right]+F\left(\gamma \rho_{0}\right)^{1 / 2} \tanh ^{-1}[\eta(\rho)]\right\},
$$

which determines the soliton phase $\Psi_{\text {sol }}(s) \equiv \Psi_{\text {sol }}[\rho(s)]$ as a functional of the intensity $\rho \equiv \rho(s)$. The phase shift across the transverse extent of the beam, denoted by $\Delta \Psi_{\text {sol }} \equiv \Psi_{\text {sol }}(+\infty)-\Psi_{\text {sol }}(-\infty)$, is given by

$$
\begin{aligned}
\Delta \Psi_{\mathrm{sol}}= & -\pi+2 \tan ^{-1}\left[\left(\frac{F}{A}\right)\left(1+\gamma \rho_{0}\right)^{1 / 2}\right] \\
& -2 F\left(\gamma \rho_{0}\right)^{1 / 2} \tanh ^{-1}\left[A\left(\gamma \rho_{0}\right)^{1 / 2}\left(1+\gamma \rho_{0}\right)^{-1 / 2}\right] .
\end{aligned}
$$

The particular case of $F=0$ corresponds to a black soliton, where there is a phase shift of $-\pi$ radians while the phase is undefined at the beam centre. For some regimes of $\gamma$, one finds that $\left|\Delta \Psi_{\text {sol }}\right|$ can exceed $\pi$ (in which case the corresponding solutions have been termed darker than black ${ }^{55}$ ).

\subsection{Intrinsic velocity}

By combining Eqs. (4.6c) and (4.7c), it can be shown that

$$
V_{0}^{2}=\frac{D \rho_{1}}{1+4 \kappa\left(\beta-D \rho_{1} / 2\right)}
$$

while, from Eq. (4.5b), one must have

$$
V_{0}^{2}=2 \frac{\beta-\chi}{1+4 \kappa \chi} .
$$




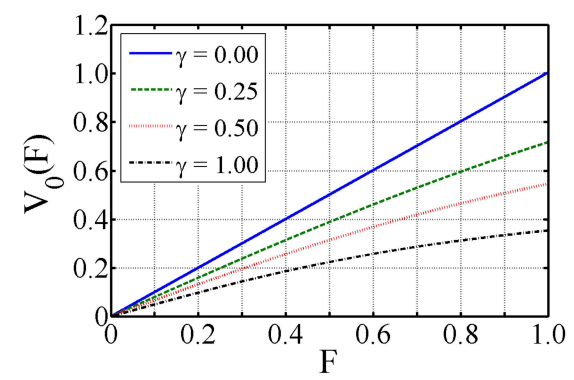

Fig. 3. Dark soliton intrinsic velocity $V_{0}$ as a function of grayness $F$ for increasing levels of saturation when the plane-wave background has intensity $\rho_{0}=1.0$ and nonparaxial parameter is set to $\kappa=1.0 \times 10^{-3}$.

Self-consistency in the solution subsequently demands that Eqs. (4.11a) and (4.11b) produce the same result for $V_{0}$, namely

$$
V_{0}=\frac{\rho_{0}^{1 / 2} F}{\left(1+\gamma \rho_{0}\right)\left(1+F^{2} \gamma \rho_{0}\right)^{1 / 2}}\left[1-\frac{2 \kappa \rho_{0}}{\left(1+\gamma \rho_{0}\right)^{2}}\left(2+\gamma \rho_{0}+\frac{F^{2}}{1+F^{2} \gamma \rho_{0}}\right)\right]^{-1 / 2}
$$

Equation (4.11c) is a key result and it completes the particular solution for which we are looking (note that the sign of $F$ controls the partiy of the soliton and also the sign of $V_{0}$ ). The effect of increasing saturation intensity on $V_{0}$ is shown in Fig. 3.

\subsection{General off-axis solution}

A more general off-axis beam can be generated by applying a coordinate rotation to the on-axis soliton. ${ }^{26}$ The new solution so derived comprises a plane wave travelling at angle $\theta=\tan ^{-1}(\sqrt{2 \kappa} V)$ with respect to the $z$ axis, and an intensity dip evolving at angle $\theta_{0}=\tan ^{-1}\left(\sqrt{2 \kappa} V_{0}\right)$ relative to that background wave. The dip, therefore, makes an angle $\theta-\theta_{0}=\tan ^{-1}(\sqrt{2 \kappa} W)$ to the $z$ axis (see Figs. 2(c) and 2(d)). The formal solution $u$ can be written as

$$
\begin{aligned}
u(\xi, \zeta)= & \rho^{1 / 2}(\xi, \zeta) \exp \left[\mp i\left\{\tan ^{-1}\left(\frac{\eta(\xi, \zeta)}{F\left(\gamma \rho_{0}\right)^{1 / 2}}\right)+F\left(\gamma \rho_{0}\right)^{1 / 2} \tanh ^{-1}[\eta(\xi, \zeta)]\right\}\right] \\
& \times \exp \left[ \pm i \sqrt{\frac{1+4 \kappa \beta}{1+2 \kappa V^{2}}}\left(-V \xi+\frac{\zeta}{2 \kappa}\right)\right] \exp \left(-i \frac{\zeta}{2 \kappa}\right),
\end{aligned}
$$

where the rotated soliton intensity distribution $\rho(\xi, \zeta)$ is obtained by solving the implicit equation 


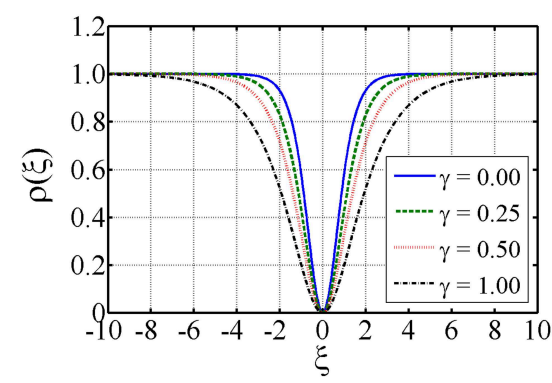

(a)

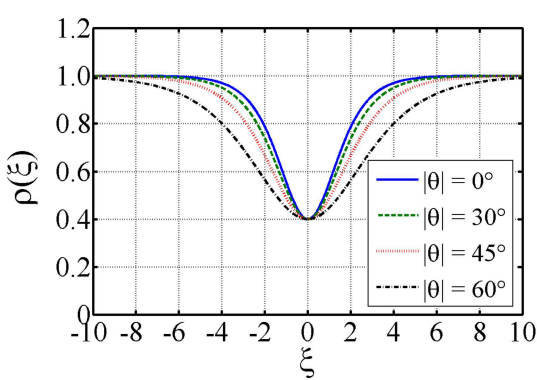

(b)

Fig. 4. (a) Changes in the intensity profile for on-axis black solitons (described by $V=0$ and $F=0=V_{0}$, respectively) as the level of saturation increases, obtained from solution (4.12b). (b) Angular beam broadening for gray solitons with $F^{2}=0.4$ and fixed saturation parameter $\gamma=0.25$. Other parameters: $\rho_{0}=1.0$ and $\kappa=1.0 \times 10^{-3}$.

$$
\begin{gathered}
\frac{1}{\left(\gamma \rho_{0}\right)^{1 / 2}} \frac{\left(1+\gamma \rho_{0}\right)^{1 / 2}}{A} \tanh ^{-1}\left[\frac{1}{\left(\gamma \rho_{0}\right)^{1 / 2}} \frac{\left(1+\gamma \rho_{0}\right)^{1 / 2}}{A} \eta(\xi, \zeta)\right]-\tanh ^{-1}[\eta(\xi, \zeta)] \\
=\frac{\gamma^{-1 / 2}}{\left(1+\gamma \rho_{0}\right)\left[1+\left(1-A^{2}\right) \gamma \rho_{0}\right]^{1 / 2}}\left(\frac{\xi+W \zeta}{\sqrt{1+2 \kappa W^{2}}}\right)
\end{gathered}
$$

(note the intensity is a symmetric function of $\xi+W \zeta$, while the phase is an antisymmetric function). The net velocity $W$ of the gray dip with respect to the $\zeta$ axis is determined by way of the geometrical result ${ }^{25,28}$

$$
W=\frac{V-V_{0}}{1+2 \kappa V V_{0}},
$$

where the intrinsic velocity $V_{0}$ of the dip relative to the plane-wave background is obtained from Eq. (4.11c). It is also straightforward to eliminate the intrinsic, transverse, and net velocities ( $V, V_{0}$ and $W$, respectively) from solution (21), so that the soliton may be expressed in terms of propagation angles $\left(\theta_{0}\right.$ and $\left.\theta\right)$ in the laboratory frame. ${ }^{25}$

Typical black soliton profiles are shown in Fig. 4(a), where an increasing level of saturation typically leads to a wider solution as might be expected on physical grounds. For fixed $\rho_{0}$, the self-defocusing effect decreases as $\gamma$ increases (i.e., as the saturation intensity drops) and hence the nonlinearity-diffraction balance necessary for supporting stationary states requires weaker diffraction (i.e., a broader beam). The geometrical (off-axis) broadening effect, as shown for gray solutions in Fig. 4(b), is a generic Helmholtz-type correction to paraxial theory which may exceed $100 \%$ even at moderate angles. ${ }^{25,28}$

\subsection{Bistability properties}

By looking at the continuum of Eqs. (4.12a) and (4.12b), it is possible to identify pairs of solutions that have the same full-width-half-maximum (FWHM), but whose 


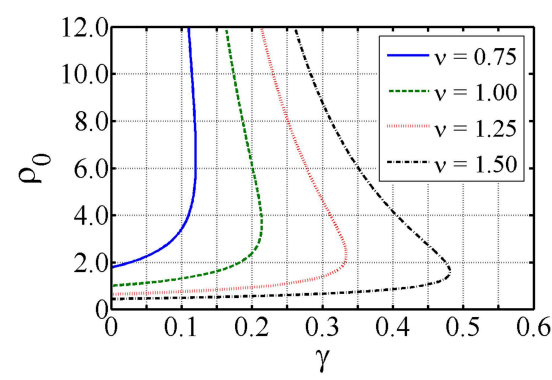

(a)

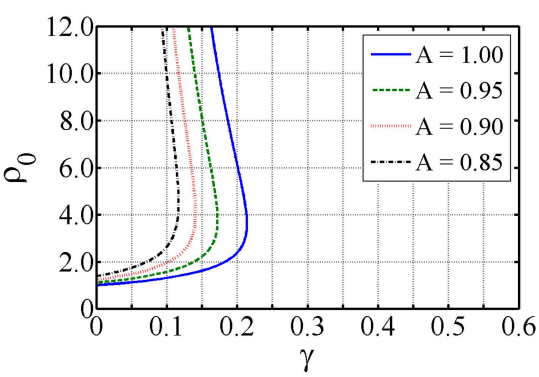

(b)

Fig. 5. Non-degenerate bistability curves for saturable dark solitons. (a) Black $(A=1)$ and (b) gray (with $\nu=1.0$ ) variants as predicted by solution (4.12a) $-(4.12 \mathrm{~b})$. Lower branches with $\gamma \rightarrow 0$ correspond to weak saturation, in which case the plane-wave intensity tends to $\rho_{0} \simeq 1 / \nu^{2} A^{2}$.

plane-wave backgrounds have different intensities (see Fig. 4). These non-degenerate soliton pairs can be obtained from the condition ${ }^{23} \rho\left(s_{1 / 2}\right)=\left(\rho_{0}+\rho_{1}\right) / 2$, where $s_{1 / 2} \equiv \nu \Delta$ and $\nu$ measures the (normalized) solution half-width in units of $\Delta \equiv$ $\operatorname{sech}^{-1}\left(2^{-1 / 2}\right) \approx 0.8814$. The implicit equation describing bistability is thus

$$
\begin{aligned}
& \frac{1}{\left(\gamma \rho_{0}\right)^{1 / 2}} \frac{\left(1+\gamma \rho_{0}\right)^{1 / 2}}{A} \tanh ^{-1}\left[\frac{1}{\left(\gamma \rho_{0}\right)^{1 / 2}}\left(\frac{1+\gamma \rho_{0}}{2-A^{2}+2\left(\gamma \rho_{0}\right)^{-1}}\right)^{1 / 2}\right] \\
& \quad-\tanh ^{-1}\left(\frac{A^{2}}{2-A^{2}+2\left(\gamma \rho_{0}\right)^{-1}}\right)^{1 / 2}=\frac{\gamma^{-1 / 2} \nu \Delta}{\left(1+\gamma \rho_{0}\right)\left[1+\left(1-A^{2}\right) \gamma \rho_{0}\right]^{1 / 2}}
\end{aligned}
$$

As $\gamma \rho_{0} \rightarrow 0$, Eq. (4.13) shows that $\rho_{0} \rightarrow 1 / \nu^{2} A^{2}$. Unlike its cubic-quintic counterpart, ${ }^{25}$ the saturable bistability condition does not appear to involve cut-off features. It is also interesting to note that Eq. (4.13) is independent of $\kappa$ (and, hence, of propagation angles) since the FWHM is defined within a frame of reference where the localized dip is on-axis. Hence, paraxial and Helmholtz bistability conditions are formally identical (as one would expect on physical grounds). Bistability curves for black solitons with various values of $\nu$, and for gray solitons with $\nu=1$, are shown in Fig. 5 .

\section{Solution Asymptotics}

We now consider the asymptotic properties of the new Helmholtz dark solitons. Two particular physical contexts are of fundamental physical importance here: firstly, the Kerr limit (which corresponds to the negligible-saturation regime of model (1.1)) and, secondly, the paraxial limit, (wherein the generic Helmholtz-type corrections can be expected to play a negligible role simultaneously). Solutions in both these regimes must necessarily be subsets of the more general predictions made by 
Eq. (3.3) - the latter corresponds to neglecting the term at $\kappa \partial_{\zeta \zeta} u$, while the former is equivalent to discarding finite- $\gamma$ considerations.

\subsection{Recovery of Kerr dark solitons}

In the limit of infinite saturation intensity, the nonlinearity in the governing equation becomes the self-defocusing Kerr-type response. One thus expects the corresponding Kerr dark soliton ${ }^{28}$ to emerge from solution (4.12a) when $\gamma \rho_{0} \rightarrow 0$ (which automatically ensures $F^{2} \gamma \rho_{0} \ll \mathcal{O}(1)$ is satisfied since $\left.0 \leq|F| \leq 1\right)$.

Binomial expansion shows that the term at $\tanh ^{-1}(\eta)$ in Eq. (4.12b) is negligible compared to the other two terms. Hence, we find that the Kerr limit corresponds to an intensity distribution described by

$$
\frac{1}{\rho_{0}^{1 / 2} A} \tanh ^{-1}\left\{\frac{\left[\rho-\rho_{0}\left(1-A^{2}\right)\right]^{1 / 2}}{\rho_{0}^{1 / 2} A}\right\} \simeq \frac{\xi+W \zeta}{\sqrt{1+2 \kappa W^{2}}} .
$$

Rearranging Eq. (5.1a) thus recovers the classic tanh-shaped dark soliton intensity profile, namely

$$
\rho(\xi, \zeta) \simeq \rho_{0}\left[1-A^{2} \operatorname{sech}^{2}\left(\rho_{0}^{1 / 2} A \frac{\xi+W \zeta}{\sqrt{1+2 \kappa W^{2}}}\right)\right] .
$$

In phase distribution (4.9c), the term at $\tanh ^{-1}(\eta)$ also tends to vanish leaving only the $\tan ^{-1}$ contribution. Hence, we find that

$$
\Psi_{\mathrm{sol}}[\rho] \simeq \mp \tan ^{-1}\left[\frac{1}{\rho_{0}^{1 / 2} F}\left(\rho-\rho_{0} F^{2}\right)^{1 / 2}\right],
$$

or equivalently,

$$
\Psi_{\mathrm{sol}}(\xi, \zeta) \simeq \mp \tan ^{-1}\left[\frac{A}{F} \tanh \left(\rho_{0}^{1 / 2} A \frac{\xi+W \zeta}{\sqrt{1+2 \kappa W^{2}}}\right)\right] .
$$

Finally, by applying the limit $\gamma \rho_{0} \rightarrow 0$ to the expression for the intrinsic velocity (see Eq. (4.11c)), one recovers the approximate result

$$
V_{0} \simeq \frac{\rho_{0}^{1 / 2} F}{\sqrt{1-2 \kappa \rho_{0}\left(2+F^{2}\right)}}
$$

and, from Eq. (3.4c), it follows that $\beta \simeq-\rho_{0} \cdot{ }^{28}$ An equivalent approach to analyzing this type of limit is to set $\gamma \rho_{0} \rightarrow 0$ in Eq. (4.7b). One then easily uncovers the familiar quadrature equation $(d \rho / d s)^{2} \simeq 4\left(\rho-\rho_{1}\right)\left(\rho_{0}-\rho\right)^{2}$, which can be integrated exactly to yield solution (5.1b).

\subsection{The paraxial limit}

When all contributions from the $\kappa \partial_{\zeta \zeta}$ operator in Eq. (3.3) are negligible simultaneously, one should be able to recover the classic paraxial dark soliton for saturable 
nonlinearity model (1.1). ${ }^{23}$ In the limit that $\kappa \rightarrow 0$ (broad beam), $\kappa \beta \rightarrow 0$ (negligible nonlinear phase shift) and $\kappa V^{2} \rightarrow 0$ (negligible propagation angle of the background wave), solution (20a) may be expressed as

$$
\begin{aligned}
u(\xi, \zeta) \simeq & \rho^{1 / 2}(\xi, \zeta) \exp \left[\mp i\left\{\tan ^{-1}\left(\frac{\eta(\xi, \zeta)}{F\left(\gamma \rho_{0}\right)^{1 / 2}}\right)+F\left(\gamma \rho_{0}\right)^{1 / 2} \tanh ^{-1}[\eta(\xi, \zeta)]\right\}\right] \\
& \times \exp \left[\mp i V \xi \pm i\left(\beta-\frac{V^{2}}{2}\right) \zeta\right] \exp \left[-i(1 \mp 1) \frac{\zeta}{2 \kappa}\right] .
\end{aligned}
$$

The forward solution maps directly onto its paraxial counterpart ${ }^{23}$ which satisfies a nonlinear Schrödinger (NLS) equation (that is, Eq. (3.3) with the term $\kappa \partial_{\zeta \zeta} u$ neglected). Moreover, the parameter $\beta$ introduced in Eq. (3.4c) can be identified with the propagation constant of the corresponding on-axis plane wave of that simpler equation.

It is also instructive to recognize that Eqs. (4.8a) and (4.9c) are formally identical in structure to the corresponding equations of paraxial theory. ${ }^{23}$ The angular distinction between Helmholtz and paraxial solutions lies in their geometric dependence on the spatial coordinates $\xi$ and $\zeta$. In the limit that $\kappa W^{2} \rightarrow 0$ (negligible propagation angle of the gray dip with respect to $z$ ), one finds that $\left(1+2 \kappa W^{2}\right)^{-1 / 2} \simeq 1-\kappa W^{2}$ so that the $\xi$ and $\zeta$ coordinates appear on the righthand side of Eq. (4.12b) as simply $\xi+W \zeta$. One can also show, from Eq. (4.12c), that the net velocity is well-approximated by $W \simeq V-V_{0}$ while the intrinsic velocity is

$$
V_{0} \simeq \frac{\rho_{0}^{1 / 2} F}{\left(1+\gamma \rho_{0}\right)\left(1+F^{2} \gamma \rho_{0}\right)^{1 / 2}} .
$$

As should be the case, one can apply the limit $\gamma \rho_{0} \rightarrow 0$ to this (exact) paraxial saturable solution and recover the Kerr dark soliton of the NLS model. ${ }^{20,21}$

The backward Helmholtz solution clearly retains a divergent $\mathcal{O}\left(\kappa^{-1}\right)$ rapidphase factor, corresponding to $\exp (-i 2 k z)$, in unscaled units. ${ }^{26}$ Hence, there can be no analogue of Helmholtz backward waves in paraxial theory (which describes propagation in a single longitudinal sense only).

\section{Dark Soliton Stability}

\subsection{Black solitons}

The stability of Helmholtz black (i.e., $F=0=V_{0}$ ) solitons may now be addressed through a suitable perturbed initial-value problem. The oblique input beam $u(\xi, 0)$ is obtained from solution (4.12a) - (4.12b) but where the broadening factor $(1+$ $\left.2 \kappa V^{2}\right)^{1 / 2}$ is omitted from the profile. So defined, one may interpret $u(\xi, 0)$ as an exact paraxial soliton being fed into Eq. (3.3) as an off-axis initial condition or, conversely (when viewed from a frame of reference where the propagating beam is on-axis), as a quasi-paraxial black soliton whose width has been reduced by $\left(1+2 \kappa V^{2}\right)^{1 / 2}$. The full width of the dark beam may then be expected to increase 


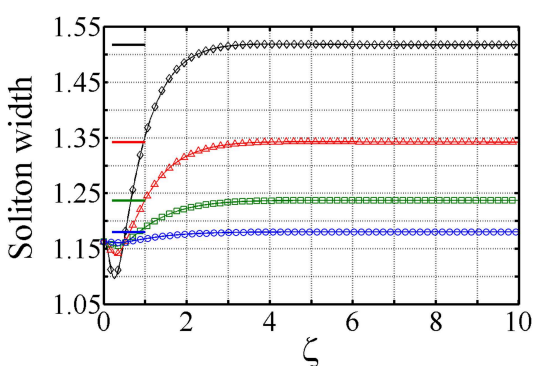

(a)

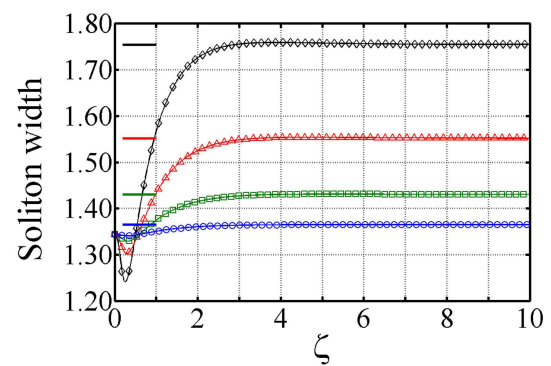

(b)

Fig. 6. Evolution of the bistable black soliton full width when the initial waveform resides on the (a) lower branch $\left(\rho_{0}=2.383\right)$ and $(\mathrm{b})$ upper branch $\left(\rho_{0}=6.167\right)$ - c.f. Fig. 5 with $\nu=1.0$. System parameters: $\gamma=0.20$ and $\kappa=1.0 \times 10^{-3}$. Blue circle: $|\theta|=10^{\circ}$. Green square: $|\theta|=20^{\circ}$. Red triangle: $|\theta|=30^{\circ}$. Black diamond: $|\theta|=40^{\circ}$. Solid bars denote asymptotic predictions.

smoothly from its value $\Lambda_{0}$ at $\zeta=0$ towards a limiting of $\Lambda_{\infty} \equiv\left(1+2 \kappa V^{2}\right)^{1 / 2} \Lambda_{0}$ as $\zeta \rightarrow \infty$.

For definiteness, we present illustrative results for the case of $\gamma=0.2$-note that the drift instability uncovered by Kivshar and Afanasjev ${ }^{56}$ for Wood et al.'s saturable nonlinearity model ${ }^{24}$ does not tend to appear for that parameter choice. Equation (4.13) predicts bistability for solutions with $\nu=1.0$ and that the planewave backgrounds must have lower- and upper-branch peak intensities of $\rho_{0} \simeq 2.383$ and $\rho_{0} \simeq 6.167$, respectively (see Fig. $5(\mathrm{a})$ ). When the nonparaxial parameter is set to $\kappa=1.0 \times 10^{-3}$, launching angles of $|\theta|=10^{\circ}, 20^{\circ}, 30^{\circ}$, and $40^{\circ}$ correspond to transverse velocities of $|V| \simeq 3.943,8.139,12.910$, and 18.763. Figure 6 shows the evolution of the beam full-width. Stationary black solitons emerge relatively quickly, with the generally adiabatic evolution allowing one to fit the numerical dataset to exact solution $(4.12 \mathrm{a})-(4.12 \mathrm{~b})$.

\subsection{Gray solitons}

The stability of gray solitons is often addressed through an integral criterion proposed by Barashenkov ${ }^{57}$ and further developed by Pelinovsky et al. ${ }^{58}$ The renormalized momentum $M_{\text {ren }}$ of a gray-type solution is defined by

$$
M_{\text {ren }} \equiv \frac{i}{2} \int_{-\infty}^{+\infty} d \xi\left(u \frac{\partial u^{*}}{\partial \xi}-u^{*} \frac{\partial u}{\partial \xi}\right)\left(1-\frac{\rho_{0}}{|u|^{2}}\right)
$$

(the formally divergent contribution to the total momentum from the on-axis background plane wave of intensity $\rho_{0}$ is subtracted, rendering $M_{\text {ren }}$ finite and generally well-behaved). Stability against small perturbations may then be expected provided

$$
\frac{d}{d V_{0}} M_{\text {ren }}\left(V_{0}\right)>0
$$

and typical renormalized-momentum curves are shown in Fig. 7. 


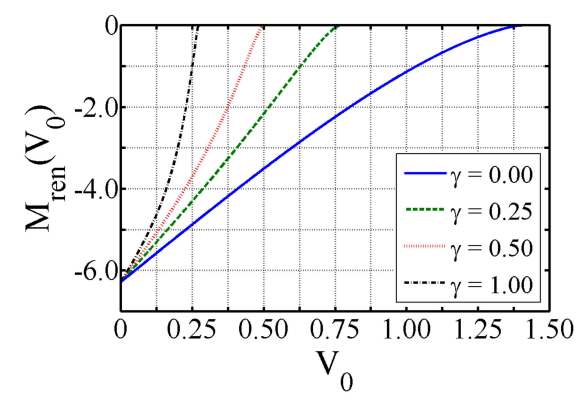

Fig. 7. Renormalized dark soliton momentum when $\rho_{0}=2.0$, computed for the contrast range $0<F \leq 1$. The gradient $d M_{\text {ren }} / d V_{0}$ is always positive for $V_{0}>0$, so that stability criterion $(6.1 \mathrm{~b})$ is always satisfied. Here, $M_{\text {ren }}$ approaches a numerical value of $-2 \pi$ as $V_{0}$ tends toward zero.

If the $\kappa$ and $\kappa \beta$ contributions from $\kappa \partial_{\zeta \zeta} u$ in Eq. (3.3) are both small, one may reasonably expect criterion (6.1b) to hold well for these quasi-paraxial Helmholtz gray solitons. That is, any meaningful prediction of stability must be effectively insensitive to the propagation angle of the plane-wave background relative to the reference (longitudinal) axis - one can always align the $z$ axis with the background wave's direction of travel and, in so doing, physical stability properties must be left unchanged.

The self-reshaping of perturbed gray solitons towards stationary states of Eq. (3.3) is similar to that shown in Fig. 6 but on a larger longitudinal scalelength. We also find that solution grayness is typically not preserved upon propagation. Simulations have shown that the contrast parameter (i.e., the beam's minimum intensity measured as a fraction of $\rho_{0}$ ) transforms according to $F \rightarrow F(\zeta)$, where $F(0)$ is specified in the initial condition and $F(\zeta)$ is obtained by fitting the numerical data to solution (4.12b). A typical example of evolving grayness is shown in Fig. 8 for $\gamma=0.2$ and $\gamma=0.1$. As $\zeta \rightarrow \infty$, the perturbed solution tends to relax back to a value of $F$ that is close to its initial value, with the relaxation process tending to take a greater distance for larger values of $\gamma$ (i.e., for lower saturation intensities).

\section{Conclusions}

We have reported the first Helmholtz dark spatial optical solitons for a saturable self-defocusing nonlinearity, complementing the bright solitons previously reported. ${ }^{41}$ In-depth analysis of these (bistable) solutions has shown that they possess the essential physical features (such as angular beam broadening) and asymptotic properties (reducing to their Kerr and paraxial counterparts in appropriate multiple limits) required of Helmholtz solitons. Theoretical and computational investigations have predicted, and confirmed, that the new solutions are robust nonlinear waves surrounded by wide basins of attraction. Perturbed black beams tend to shed low-amplitude radiation as they evolve smoothly towards stationary states 


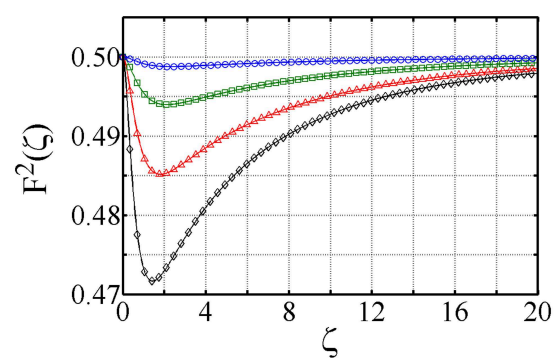

(a)

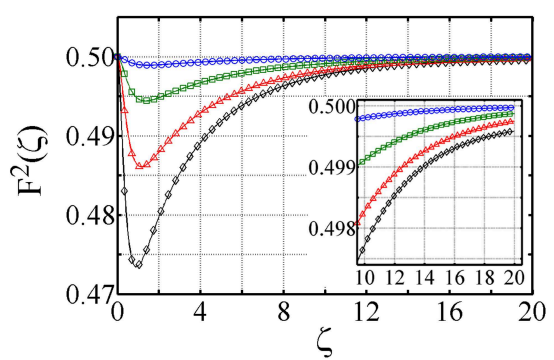

(b)

Fig. 8. Evolution of the soliton grayness parameter for $\rho_{0}=2.0$ when (a) $\gamma=0.2$ and (b) $\gamma=0.1$ (inset: expanding vertical scale so as the curves may be resolved) for nonparaxial parameter $\kappa=1.0 \times 10^{-3}$. Blue circle: $|\theta|=10^{\circ}$. Green square: $|\theta|=20^{\circ}$. Red triangle: $|\theta|=30^{\circ}$. Black diamond: $|\theta|=40^{\circ}$.

of Eq. (3.3), and relatively quickly. Gray beams share similar properties but the relaxation process is noticeably slower.

From a strictly mathematical perspective, it would be profitable to see if other ways of seeking broad solution classes ${ }^{29}$ - e.g., tanh, elliptic, and exponential expansion methods and their extensions, $F$ - and $G^{\prime} / G$-expansions, and homogeneousbalance methods - can also be applied to Helmholtz-type governing equations. To date, we have focused primarily on 'blunt-instrument' ansatz approach (where a good guess at a trial solution can be made) and the more elegant direct-integration technique (as deployed in this paper), both combined with geometrical transformations. These two methods have shown themselves to be powerful tools for identifying solitary-type excitations in spatially-symmetric equations and where the nonlinearity is homogeneous (e.g., does not contain derivatives).

The research presented here completes our analysis of exact scalar spatial solitons for complementary saturable nonlinearities. ${ }^{41}$ Knowledge about the mathematical form and physical stability properties of these solutions opens up the possibility of modelling oblique beam propagation in a wide range of device architectures. For instance, the refraction of bright ${ }^{42,43}$ and dark ${ }^{44,45}$ beams at a single interface are problems of pivotal importance where non-trivial angular effects are the rule rather than the exception. These research areas can now be extended beyond Kerr media to configurations involving both cubic-quintic ${ }^{25,59}$ and, crucially, saturable-type materials. One can also revisit periodic (e.g., coupled-waveguide) systems that have historically been analyzed only for paraxial diffraction. Headon $^{60,61}$ and side-coupling ${ }^{62,63}$ geometries are both of interest in Helmholtz-angular contexts. ${ }^{64}$

\section{Acknowledgement}

This work was supported by the Engineering and Physical Sciences Research Council (EPSRC), grant no. EP/H011595/1, and the University of Salford. 


\section{References}

1. A. E. Siegman, Lasers (University Science Books, 1986).

2. P. Mandel, Theoretical Problems in Cavity Nonlinear Optics (Cambridge University Press, 1997).

3. P. Roussignol, D. Ricard, J. Lukasik, and C. Flytzanis, New results on optical phase conjugation in semiconductor-doped glasses, J. Opt. Soc. Am. B 4 (1987) $5-13$.

4. J.-L. Coutaz and M. Kull, Saturation of the nonlinear index of refraction in semconductor-doped glass, J. Opt. Soc. Am. B 8 (1991) 95-98.

5. T. Catunda, and L. A. Cury, Transverse self-phase modulation in ruby and $\mathrm{GdAlO}_{3}: \mathrm{Cr}^{+3}$ crystals, J. Opt. Soc. Am. B 7 (1990) 1445-1455.

6. Q. Wang Song, X.-M. Wang, R. R. Birge, J. D. Downie, D. Timucin, and C. Gary, Propagation of a Gaussian beam in a bateriorhodopsin film, J. Opt. Soc. Am. B 15 (1998) 1602-1609.

7. L. Demenicis, A. S. L. Gomes, D. V. Petrov, C. B. de Arajo, C. P. de Melo, C. G. dos Santos, and R. Souto-Maior, Saturation effects in the nonlinear-optical susceptibility of poly(3-hexadecylthiophene), J. Opt. Soc. Am. B 14 (1997) 609614.

8. S. Bian, J. Frejlich, and K. H. Ringhofer, Photorefractive saturable Kerr-type nonlinearity in photovoltaic crystals, Phys. Rev. Lett. 78 (1997) 4035-4038.

9. D. N. Christodoulides and M. I. Carcalho, Bright, dark, and gray spatial soliton states in photorefractive media, J. Opt. Soc. Am. B 12 (1995) 1628-1633.

10. G. F. Calvo, J. Belmonte-Beitia, and V. M. Pérez-Garca, Exact bright and dark spatial soliton solutions in saturable nonlinear media, Chaos, Solitons and Fractals 41 1791-1798.

11. S. Gatz and J. Herrmann, Propagation of optical beams and the properties of two-dimensional spatial solitons in media with a local saturable nonlinear refractive index, J. Opt. Soc. Am. B 14 (1997) 1795-1806.

12. Y. Chen, Dark spatial solitons in non-Kerr-law media, Phys. Rev. A 45 (1992) $5215-5219$.

13. Y. Chen, Self-trapped light in saturable nonlinear media, Opt. Lett. 16 (1991) $4-6$.

14. B. V. Gisin and B. A. Malomed, Subwavelength spatial solitons in optical media with non-Kerr nonlinearities, J. Opt. A: Pure Appl. Opt. 3 (2001) 284-290.

15. B. V. Gisin and B. A. Malomed, One- and two-dimensional subwavelength solitons in saturable media, J. Opt. Soc. Am. B 18 (2001) 1356-1361.

16. N. N. Rosanov, V. E. Semenov, and N. V. Vyssotina, J. Opt. B: Quantum Semiclass. Opt. 3 (2001) S96-S99.

17. S. Gatz and J. Herrmann, Soliton propagation and soliton collision in doubledoped fibers with a non-Kerr-like nonlinear refractive-index change, Opt. Lett. 17 (1992) 484-486.

18. S. Gatz and J. Herrmann, Soliton propagation in materials with saturable non- 
linearity, J. Opt. Soc. Am. B 8 (1991) 2296-2302.

19. J. Herrmann, Propagation of ultrashort light pulses in fibers with saturable nonlinearity in the normal-dispersion region, J. Opt. Soc. Am. B 8 (1991) 15071511.

20. Y. S. Kivshar, Bright and dark spatial solitons in non-Kerr media, Opt. Quantum Electron. 30 (1998) 571-614.

21. Y. S. Kivshar and B. Luther-Davies, Dark optical solitons: physics and applications, Phys. Rep. 298 (1998) 81-197.

22. W. Krolikowski and B. Luther-Davies, Analytic solution for soliton propagation in a nonlinear saturable medium, Opt. Lett. 17 (1992) 1414-1416.

23. W. Krolikowski and B. Luther-Davies, Dark optical solitons in saturable nonlinear media, Opt. Lett. 18 (1993) 188-190.

24. V. W. Wood, E. D. Evans, and R. P. Kenan, Soluble saturable refractive-index nonlinearity model, Opt. Commun. 69 (1988) 156-160.

25. J. M. Christian, G. S. McDonald, and P. Chamorro-Posada, Bistable dark solitons of a cubic-quintic Helmholtz equation, Phys. Rev. A 81 (2010) 053831.

26. P. Chamorro-Posada, G. S. McDonald, and G. H. C. New, Exact soliton solutions of the nonlinear Helmholtz equation: communication J. Opt. Soc. Am. B 19 (2002) 1216-1217.

27. M. Crosta, A. Fratalocci, and S. Trillo, Bistability and instability of darkantidark solitons in the cubic-quintic nonlinear Schrödinger equation, Phys. Rev. A 84 (2011) 063809.

28. P. Chamorro-Posada and G. S. McDonald, Helmholtz dark solitons, Opt. Lett. 28 (2003) 825-827.

29. L. Ling-Xiao and W. Ming-Liang, The $\left(G^{\prime} / G\right)$ expansion method and travelling wave solutions for a higher-order nonlinear Schrödinger equation, Appl. Math. Comp. 208 (2009) 440-445.

30. Y. Xu, J. Vega-Guzman, D. Milovic, M. Mirzazadeh, M. Eslam, M. F. Mahmood, A. Biswas, and M. Belic, Bright and exotic solitons in optical metamaterials by semi-inverse variational principle, J. Nonlin. Opt. Phys. Mat. 24 (2015) 1550042 .

31. M. Mirzazadeh, M. Eslami, Q. Zhou, M. F. Mahmood, E. Zerrad, A. Biswas, and M. Belic, Optical solitons in nonlinear directional couplers with $G^{\prime} / G$-expansion scheme, J. Nonlin. Opt. Phys. Mat. 24 (2015) 1550017.

32. R. Parada-Algonso, L. C. Gomez-Pavon, A. Luis-Ramos, and J. M. MunozPacheco, Self-compression of coupled cnoidal waves, J. Nonlin. Opt. Phys. Mat. 24 (2015) 1550010.

33. A. Saini, V. M. Vyas, T. S. Raju, S. N. Pandev, P. K. Panigrahi, Super and subluminal propagation in nonlinear Schrodinger equation model with selfsteepening and self-frequency shift, J. Nonlin. Opt. Phys. Mat. 24 (2015) 1550033.

34. R. Pal, A. Goval, S. Loomba, T. S. Raju, and C. N. Kumar, Compression of optical similaritons induced by cubic-quintic nonlinear media in a graded-index 
waveguide, J. Nonlin. Opt. Phys. Mat. 25 (2016) 1650033.

35. Q. Jiang, Y. Su, H. Nie, Z. Ma, and Y. Li, New type gray spatial solitons in twophoton photorefractive media with both the linear and quadratic electro-optic effects, J. Nonlin. Opt. Phys. Mat. 26 (2017) 1750006.

36. A. Alberucci, G. Assanto, J. M. L. MacNeil, and N. F. Smyth, Nematic liquid crystals: An excellent playground for nonlocal nonlinear light localization in soft matter, J. Nonlin. Opt. Phys. Mat. 23 (2014) 1450046.

37. M. Ekici, M. Mirzazadeh, A. Sonmezoglu, M. Z. Ullah, Q. Zhou, S. P. Moshokoa, A. Biswas, and M. Belic, Nematicons in liquid crystals by extended trial equation method, J. Nonlin. Opt. Phys. Mat. 26 (2017) 1750005.

38. P. Panayotaros, Shelf solutions and dispersive shocks in a discrete NLS equation: Effects of nonlocality, J. Nonlin. Opt. Phys. Mat. 25 (2016) 1650045.

39. P. Chamorro-Posada and G. S. McDonald, Helmholtz non-paraxial beam propagation method: An assessment, J. Nonlin. Opt. Phys. Mat. 23 (2009) 1450040.

40. P. Chamorro-Posada and G. S. McDonald, Spatial Kerr soliton collisions at arbitrary angles, Phys. Rev. E $\mathbf{7 4}$ (2006) 036609.

41. J. M. Christian, G. S. McDonald, and P. Chamorro-Posada, Bistable Helmholtz bright solitons in saturable materials, J. Opt. Soc. Am. B 26 (2009) 2323-2330.

42. J. Sánchez-Curto, P. Chamorro-Posada, and G. S. McDonald, Helmholtz solitons at nonlinear interfaces, Opt. Lett. 32 (2007) 1126-1128.

43. J. Sánchez-Curto, P. Chamorro-Posada, and G. S. McDonald, Helmholtz bright and black soliton splitting at nonlinear interfaces, Phys. Rev. A 85 (2012) 013836.

44. J. Sánchez-Curto, P. Chamorro-Posada, and G. S. McDonald, Black and gray Helmholtz-Kerr soliton refraction, Phys. Rev. A 83 (2012) 013828.

45. J. Sánchez-Curto, P. Chamorro-Posada, and G. S. McDonald, Dark solitons at nonlinear interfaces, Opt. Lett. 35 (2010) 1347-1349.

46. M. Lax, W. H. Louisell, and W. B. McKnight, From Maxwell to paraxial wave optics, Phys. Rev. A 11 (1975) 1365-1370.

47. S. Chi and Q. Guo, Vector theory of self-focusing of an optical beam in Kerr media, Opt. Lett. 20 (1995) 1598-1600.

48. B. Crosignani, A. Yariv, and S. Mookherjea, Nonparaxial spatial solitons and propagation-invariant pattern solutions in optical Kerr media, Opt. Lett. 29 (2004) 1254-1257.

49. A. Ciattoni, B. Crosignani, S. Mookherjea, and A. Yariv, Nonparaxial dark solitons in optical Kerr media, Opt. Lett. 30 (2005) 516-518.

50. M. D. Feit and J. A. Fleck, Beam nonparaxiality, filament formation, and beam breakup in the self-focusing of optical beams, J. Opt. Soc. Am. B 5 (1988) 633640 .

51. A. P. Sheppard and M. Haelterman, Nonparaxiality stabilizes three-dimensional soliton beams in Kerr media, Opt. Lett. 23 (1998) 1820-1822.

52. T. A. Laine and A. T. Friberg, Self-guided waves and exact solutions of the nonlinear Helmholtz equation, J. Opt. Soc. Am. B 17 (2000) 751-757. 
53. J. M. Christian, G. S. McDonald, and P. Chamorro-Posada, Helmholtz bright and boundary solitons, J. Phys. A: Math. Theor. 40 (2007) 1545-1560.

54. P. Chamorro-Posada, G. S. McDonald, and G. H. C. New, Non-paraxial beam propagation methods, Opt. Commun. 192 (2001) 1-12.

55. W. Krolikowski, N. Akhmediev, and B. Luther-Davies, Darker-than-black solitons: dark solitons with total phase shift greater than $\pi$, Phys. Rev. E 48 (1993) 3980-3987.

56. Y. S. Kivshar and V. V. Afanasjev, Drift instability of dark solitons in saturable media, Opt. Lett. 21 (1996) 1135-1137.

57. I. V. Barashenkov, Stability criterion for dark solitons, Phys. Rev. Lett. 77 (1996) 1193-1197.

58. D. E. Pelinovsky, Y. S. Kivshar, and V. V. Afanasjev, Instability-induced dynamics of dark solitons, Phys. Rev. E 54 (1996) 2015-2032.

59. J. M. Christian, G. S. McDonald, and P. Chamorro-Posada, Bistable Helmholtz solitons in cubic-quintic materials, Phys. Rev. A 76 (2007) 033833.

60. D. Neshev, A. A. Sukhorukov, B. Hanna, W. Krolikowski, and Y. S. Kivshar, Controlled generation and steering of spatial gap solitons, Phys. Rev. Lett. 93 (2004) 083905.

61. A. A. Sukhorukov, D. Neshev, W. Krolikowski, and Y. S. Kivshar, Nonlinear Bloch-wave interaction and Bragg scattering in optically induced lattices, Phys. Rev. Lett. 92 (2004) 093901.

62. D. Mandelik, R. Morandotti, J. S. Aitchison, and Y. Silberberg, Gap solitons in waveguide arrays, Phys. Rev. Lett. 92 (2004) 093904.

63. D. Mandelik, H. S. Eisenberg, Y. Silberberg, R. Morandotti, and J. S. Aitchison, Band-gap structure of waveguide arrays and excitation of Floquet-Bloch solitons, Phys. Rev. Lett. 90 (2003) 053902.

64. E. A. McCoy, New Modelling Applications for Helmholtz Soliton Theory: From Single Interfaces to Waveguide Arrays (Ph.D. dissertation, University of Salford, U.K., 2014). 